\title{
Trauma in pregnant women: an experience from a level 1 trauma center
}

\author{
Mishal Gillani ${ }^{1 *}$, Sabah Uddin Saqib², Russell Seth Martins ${ }^{1}$, Hasnain Zafar ${ }^{2}$ \\ 'Medical College, Aga Khan University Hospital, Stadium Road, Karachi, 74800, Pakistan \\ 2Department of Surgery, Aga Khan University Hospital, Stadium Road, Karachi, 74800, Pakistan
}

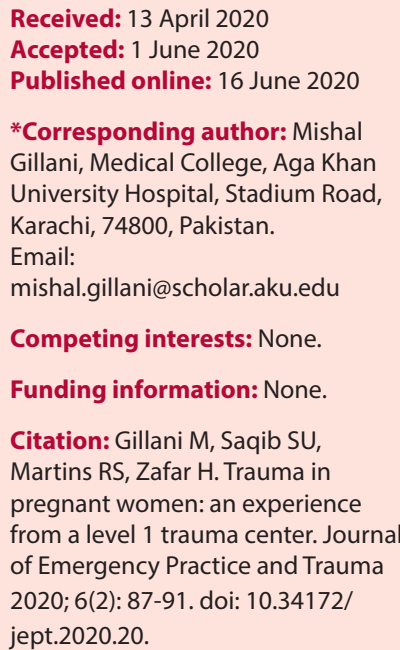

\begin{abstract}
Objective: Trauma is the significant non-obstetrical cause of maternal mortality in women aged 35 years or younger. It is expected to complicate around 1 in 12 pregnancies and accounts for $46 \%$ of such deaths. In this study, we present our experience of trauma during pregnancy at a tertiary care hospital in Karachi.

Methods: A standardized form was used to extract data from online records for all pregnant women who presented with traumatic injuries to the Aga Khan University Hospital from 2014 to 2019. Analysis was performed using SPSS v. 21. Chi-squared tests were performed for comparison of categorical data.

Results: A total of 48 pregnant females with a mean age of $28.80 \pm 6.50$ years were included in this study. Road traffic accidents (RTA) accounted for the commonest cause of injury in the first $(66.7 \%)$ and second (65\%) trimesters. However, fall $(45.5 \%)$ followed by RTA $(27.3 \%)$ was the most frequent mechanism of injury during the third trimester. Gunshot injuries were seen in 4 patients only. Overall, fetal ultrasound was the most commonly performed imaging (87.5\%) followed by limb X-ray and focused abdominal sonography for trauma (FAST) ultrasound. Out of total, $52.1 \%$ of the patients were surgically managed. Fetal demise in utero and spontaneous abortion was reported in 2 patients only. Conclusion: Road traffic accidents and falls comprise a significant burden of maternal and fetal morbidity and mortality. In terms of prevention, several policies and interventions at the government level need to be introduced in order to reduce the incidence of traumatic events.

Keywords: Pregnancy, Trauma, Developing country, Road traffic accidents, Pakistan
\end{abstract}

\section{Introduction}

Trauma is the significant non-obstetrical cause of maternal mortality in women aged 35 years or younger (1). It is expected to complicate around 1 in 12 pregnancies (2). The risk of fetal mortality has been reported to be greater than that of maternal mortality. Notable causes accounting for high fetal mortality are placental abruption, maternal shock, uterine rupture, disseminated intravascular shock, direct fetal injury and pre-term delivery (3).

The mechanisms of injury and mortality are classified into various types, including falls, burns, assaults, blunt and penetrating trauma (4). Motor vehicle collisions account for around $80 \%$ of all major traumatic incidents during pregnancy, followed by fall and assault (5). Literature shows that every 1 in 3 pregnant women inflicted with trauma is expected to deliver when hospitalized (6).

Acquaintance with common mechanisms of injuries and their management will allow physicians to provide appropriate treatment to pregnant women. In this study, we present our experience of trauma during pregnancy at a tertiary care hospital in Karachi, Hospital.

\section{Methods}

This cross-sectional study was conducted at the Aga Khan University (AKU) Hospital's Department of Surgery over a period of 5 years from 2014 to 2019. Participants were selected using non-probability consecutive sampling. The study protocol was approved by the institutional review board of AKU Hospital.

Data were collected from patients' charts and medical records by the research team, using a standardized form which included the following sections:

- Patient's characteristics: Age (years) and information regarding the weeks of pregnancy were documented.

- Mechanism of trauma: Information regarding the site of injury and mechanism, whether it was blunt or penetrating or both was recorded.

- Management and outcomes: Data regarding the 
management approach including the type of imaging used was documented. Furthermore, fetal and maternal complications together with the outcomes were also reported.

Data were analyzed using IBM SPSS (Statistical Package for Social Sciences) version 23.0. Continuous data were presented using means and standard deviations, whereas categorical data were presented using numbers and percentages. Chi-squared tests were performed for comparison of categorical data. $P$ value $<0.05$ considered as significant.

\section{Results}

A total of 48 pregnant females with the mean age of 28.80 \pm 6.50 years were included in this study. Around $58.3 \%$ of patients presented directly to the emergency room (ER), while others came to the consultant clinics. Majority of the cohort was treated under the services of obstetrics/ gynecology (41.7\%), followed by orthopedics (33.3\%) and general surgery $(10.4 \%)$. At time of presentation, most females were in the third trimester of pregnancy (45.8\%). The mean length of stay was $3.35 \pm 3.19$ days. Findings are shown in Table 1.

Overall, road traffic accidents (RTA) were the most common mechanism of injury (47.9\%), followed by fall (31.3\%). Other mechanisms of injury included cutting instrument injury, domestic violence and penetrating injuries. The most common site of injury was upper limb (31.3\%), followed by lower limb (25.0\%), abdomen $(16.7 \%)$ and spine $(8.3 \%)$, respectively.

RTA accounted for the most common cause of injury in the first $(66.7 \%)$ and second (65\%) trimesters. However, during the third trimester, the most frequent mechanism

Table 1. Maternal and fetal characteristics $(n=48)$

\begin{tabular}{lc}
\hline Variables & Mean \pm SD/ No.(\%) \\
\hline Age $(y)$ & $28.80 \pm 6.50$ \\
\hline Length of stay (days) & $3.35 \pm 3.19$ \\
Admission source & $28(58.3)$ \\
Emergency room & $20(41.7)$ \\
Consultant clinics & \\
Admitting department & $20(41.7)$ \\
Obstetrics/gynecology & $16(33.3)$ \\
Orthopedic surgery & $5(10.4)$ \\
General surgery & $3(6.3)$ \\
Plastics & $2(4.2)$ \\
Neurosurgery & $2(4.2)$ \\
Medicine $_{\text {Trimester }}$ & \\
$1^{\text {st }}$ & $22(12.5)$ \\
$2^{\text {nd }}$ & $20(41.7)$ \\
$3^{\text {rd }}$ & $2(45.8)$ \\
\hline
\end{tabular}

of injury was fall (45.5\%), followed by RTA (27.3\%). Gunshot injuries were seen in 4 patients only. The upper limb was the commonest site of injury in all three trimesters. In the second and third trimesters, traumatic injuries also commonly involved abdomen and lower limb. Findings are depicted in Table 2.

Overall, fetal ultrasound was the most commonly performed imaging (87.5\%), followed by limb X-ray and focused abdominal sonography for trauma (FAST) ultrasound.

In $1^{\text {st }}$ trimester patients, limb X-ray was done most commonly (83.3\%), followed by chest X-ray (50\%) and computed tomography (CT) scan (33.3\%). During the second trimester, fetal ultrasound (95\%) and FAST ultrasound (55\%) were the most used imaging modality. Additionally, in the second trimester, limb X-ray (35\%), chest X-ray (35\%) and CT scan (25\%) were also done in some patients. In the third trimester patients, fetal ultrasound was done most frequently (77.3\%). Furthermore, limb X-ray and FAST ultrasound also remained a popular choice of imaging in third trimester. Findings are presented in Table 2.

Out of total, $52.1 \%$ of the patients were surgically managed. However, caesarean section (C-section) was done in $10.4 \%$ of patients only. Overall, open reduction for fracture repair (31.3\%) was the most frequently performed surgical procedure followed by wound debridement and superficial repair (18.8\%). Most procedures were done under general anesthesia $(41.7 \%)$, while local anesthesia was given in $5(10.4 \%)$ cases. Blood products were used in 2 patients. During the first, second and third trimester, $50 \%, 40 \%$ and $63.6 \%$, of the patients were managed surgically. Open reduction for fracture repair was the most commonly performed surgical procedure in all three trimesters. All other frequently performed procedures are listed in Table 2. There was no harm to fetus in $95.8 \%$ of the cases and the baby remained healthy. Fetal demise in utero and spontaneous abortion were reported in 2 patients only. A total of 47 (97.9\%) patients had a healthy recovery, while paraplegia occurred in 1 case. All findings are shown in Table 2.

\section{Discussion}

The mean age of the patients in our study was $28.80 \pm$ 6.50 years. Around $58.3 \%$ of patients presented directly to the emergency department. Our results showed that most patients $(41.7 \%)$ were treated under the services of obstetrics/gynecology, followed by orthopedic surgery and general surgery. At time of presentation, most females in our study were in the third trimester of pregnancy (45.8\%). A previous study by Weiss et al showed that the mean age of pregnant women who came to the emergency department for treatment was 24.6 years (5) which is close to the mean age in our study. Likewise, in another study, the mean age of sample was $26.72 \pm 6.29$ years, with majority being in the third trimester (7). A previous study 
Table 2. Commonest injury patterns, imaging modalities and management according to trimesters

\begin{tabular}{|c|c|c|}
\hline $1^{\text {st }}$ Trimester $(n=6)$, No. $(\%)$ & $2^{\text {nd }}$ Trimester $(n=20)$, No. $(\%)$ & $3^{\text {rd }}$ Trimester $(n=22)$, No. (\%) \\
\hline \multicolumn{3}{|c|}{ Commonest mechanism of injury $(P$ value $=0.120)$} \\
\hline RTA 4 (66.7) & RTA $13(65.0)$ & Fall $10(45.5)$ \\
\hline Gunshot 1 (16.7) & Fall $5(25.0)$ & RTA 6 (27.3) \\
\hline \multirow[t]{3}{*}{ Others 1 (16.7) } & Gunshot $1(5.0)$ & Gunshot 2 (9.1) \\
\hline & Others $1(5.0)$ & Domestic Violence 1 (4.5) \\
\hline & & Others 3 (13.6) \\
\hline \multicolumn{3}{|c|}{ Commonest site of injury $(P$ value $=0.214)$} \\
\hline Upper Limb 2 (33.3) & Upper Limb 5 (25.0) & Upper Limb 8 (36.4) \\
\hline Spine 2 (33.3) & Abdomen 5 (25.0) & Lower Limb 7 (31.8) \\
\hline Lower Limb 1 (16.7) & Lower Limb 4 (20.0) & Abdomen 3 (13.6) \\
\hline Chest 1 (16.7) & Spine 2 (10.0) & Head 1 (4.5) \\
\hline \multirow[t]{4}{*}{ Pelvis 1 (16.7) } & Head $1(5.0)$ & Others 3 (13.6) \\
\hline & Chest 1 (5.0) & \\
\hline & Pelvis 1 (5.0) & \\
\hline & Others $1(5.0)$ & \\
\hline \multicolumn{3}{|c|}{ Commonest imaging modality used $(P$ value $=0.007)$} \\
\hline Limb X-ray 5 (83.3) & Fetal Ultrasound 19 (95.0) & Fetal Ultrasound 17 (77.3) \\
\hline Chest X-ray $3(50.0)^{*}$ & Fast Ultrasound $11(55.0)^{*}$ & Limb X-ray 13 (59.1) \\
\hline CT Scan 2 (33.3) & Limb X-ray 7 (35.0) & Fast Ultrasound $4(18.2)^{*}$ \\
\hline Pelvic X-ray 2 (33.3) & Chest X-ray $7(35.0)^{*}$ & Chest X-ray $2(9.1)^{*}$ \\
\hline \multirow[t]{3}{*}{ Fast Ultrasound $1(16.7)^{*}$} & CT Scan 5 (25.0) & Pelvic X-ray 2 (9.1) \\
\hline & Pelvic X-ray 3 (15.0) & CT Scan 1 (4.5) \\
\hline & & MRI Head 1 (4.5) \\
\hline \multicolumn{3}{|c|}{ Management $(P$ value $=0.304)$} \\
\hline Conservative 3 (50.0) & Conservative $12(60.0)$ & Conservative 8 (36.4) \\
\hline Surgical $3(50.0)$ & Surgical 8 (40.0) & Surgical 14 (63.6) \\
\hline \multicolumn{3}{|c|}{ Commonest procedure among those surgically managed $(P$ value $=0.569)$} \\
\hline Open reduction internal fixation 3 (100) & Open reduction internal fixation 6 (75.0) & Open reduction internal fixation 6 (42.9) \\
\hline Spine fixation 1 (33.3) & Debridement and Repair 3 (37.5) & Debridement and Repair 5 (35.7) \\
\hline \multirow[t]{4}{*}{ Debridement and Repair 1 (33.3) } & C-section 1 (12.5) & C-section 4 (28.6) \\
\hline & Skin Grafting 1 (12.5) & Neurosurgical Decompression 1 (7.1) \\
\hline & Ligament Repair 1 (12.5) & Tracheostomy 1 (7.1) \\
\hline & Spine Fixation 1 (12.5) & \\
\hline
\end{tabular}

by Weiss et al reported that the average gestational week of injury was 21 weeks (5).

Overall, our data revealed that RTA was the most common mechanism of injury (47.9\%), followed by fall and gunshot injuries. According to a review article, blunt trauma was the most common mechanism of injury in $69 \%$ of pregnant women in comparison with $1.5 \%$ who experienced penetrating trauma (8). Furthermore, among the causes of blunt trauma, motor-vehicle collisions accounted for the commonest cause of injury followed by falls, assault and gunshot wounds (8).

According to our study, the most common site of injury was the upper limb (31.3\%), followed by the lower limb, abdomen and spine. Similarly, in a review conducted by Zangene et al, the most common site of injuries was lower extremities, in $67 \%$ of patients (9). A study focusing on the link of maternal fractures and perinatal outcomes showed that $44 \%$ of pregnant women who experienced motor vehicle collisions had fractures. High mortality and morbidity were seen in patients with pelvic fractures (10). In our study, RTA accounted for the most frequent cause of injury in the first and second trimester. In contrast, falls were most frequently seen in patients in the third trimester. Previous literature shows that falls account for the second most frequent cause of traumatic injuries during pregnancy, particularly during the second and third trimester due to an increase in weight and changes in the centre of gravity (8).

Trauma due to domestic violence was seen in only 1 patient. A study conducted in America showed that the prevalence of interpersonal violence (IPV) during pregnancy spans between 1-20\%, with the domestic 
partner being the abuser in the most of the cases (11). Furthermore, a study from Iran showed an incidence of $47 \%$ of domestic violence in pregnant women, mainly during the second trimester (12). This shows how IPV may be more prevalent in the developing world. Although only one female presented with trauma due to IPV, it is possible that this number is low due to the mass underreporting of IPV in Pakistan (13). Most injuries caused by IPV are confined to the soft tissues and the torso, excluding the abdomen. If the abdomen is involved there is a high incidence of antepartum haemorrhage with further complexities (8).

On the whole, in the present study, fetal ultrasound was the most commonly performed imaging (87.5\%), followed by limb X-ray and FAST ultrasound. Imaging during pregnancy and the harmful effects that radiation poses on fetus remain debatable topics. However, if radiographic imaging is justified medically then the indications are similar as for any other trauma inflicted on a non-pregnant female. The imaging does not need to be postponed if the benefits dominate over the risks (8). Additionally, literature suggests that required imaging should be performed despite the status of the pregnancy. As the major part of human data on radiation exposure is not according to the doses of radiation used during normal radiographic imaging, hence it is stated that there is no additional risk of congenital malformations or miscarriages if the fetus is exposed to less than 5-10 rad (14).

Moreover, gestational age plays a significant role in determining the effect of radiation on fetus, with the most susceptible time being the first 16 weeks of pregnancy as most of the embryogenesis occurs during this time (14). CT scan can reveal injuries but is only indicated if the patient is stable hemodynamically. Overall, FAST ultrasound is considered the gold standard as it is noninvasive, safe, and can identify fetal well-being (8).

In conclusion, it is better to limit the number of radiological investigations in order to establish the diagnosis. Also, it is encouraged to cover the abdomen with lead apron when the imaging does not involve the abdomen.

In our sample, $52.1 \%$ of the patients were surgically managed. However, C-section was done in $10.4 \%$ of patients only. Beyond the gestational age of 24 weeks, emergency C-section has a beneficial impact on maternal or fetal outcome (14). Additionally, sometimes clinical examination is not enough to determine the degree of uterine damage and injury to nearby organs, hence in some cases exploratory surgery needs to be performed (14). Moreover, if a non-obstetric surgery needs to be done, it is vital to maintain uterine perfusion, maternal oxygenation and circulating blood volume (14).

Our study revealed no maternal mortality, while fetal demise in-utero and spontaneous abortion were reported in 2 patients only. A fetal death certificate study revealed the calculated fetal mortality resulting from trauma during pregnancy to be 2.3 per 100000 live births (15). Risk of maternal trauma changes with gestational age largely due to the growing size of fetus and uterus. Maternal death rates have been noted to be as high as $10-15 \%$ during the first trimester, $32-40 \%$ during the second trimester, and 50\%-54\% during the third trimester (16). Furthermore, a study stated that women experiencing an injury during the first trimester are at a greater risk of complications and pre-term birth as compared to women injured during the third trimester (5). A study by Weiss et al. showed that out of all traumatically injured pregnant women who presented to the ER and experienced a live birth, $97.8 \%$ were outpatients and 2.2\% were hospitalized (5).

Although the literature on maternal trauma is quite comprehensive, there is scarce data from developing countries reflecting the impact of trauma on maternal and fetal outcomes. The optimal means of managing pregnant women suffering from trauma remains a topic warranting additional exploration. Pattern of injuries and facilities available to manage patients differ in developing countries like Pakistan. Enforcement of traffic safety rules and improvement of traffic and road infrastructure may help to reduce RTAs, which comprise a significant source of trauma. Moreover, mass education and awareness campaigns may be used to improve maternal behaviour during pregnancy, which could help prevent trauma due to falls. Additionally, since many buildings lack elevators, installation of elevators could also help reduce falls. Last but not least, it is also important to perform screening for domestic violence during pre-natal hospital visits if possible, as IPV remains a grossly under-reported phenomenon in Pakistan.

\section{Limitations}

Although this is a single-centre study, The Aga Khan Hospital has one of the few Level 1 Trauma Facilities in Karachi and receives a major load of the city's trauma cases. However, additional studies using multiple centres and larger populations may add further insight to the subject.

\section{Conclusion}

RTA and falls comprise a significant burden of maternal and fetal morbidity and mortality. In order to improve outcomes, it is important to be acquainted with the spectrum of injuries, diagnostic imaging approach and the necessary management approach involved in trauma during pregnancy. In terms of prevention, several policies and interventions at the government level need to be introduced in order to reduce the incidence of traumatic events. The findings of our research offer the initial foundation for the development of specific guidelines for the prevention and management of trauma in pregnancy.

\section{Authors' contributions}

MG was a major contributor in writing the manuscript 
and performed the data analysis. SS contributed in the manuscript writing. RSM helped with manuscript writing and data collection. HZ helped with the project design and provided a critical review. All authors read and approved the final manuscript.

\section{Ethics issues}

This study received ethical approval from the Institutional Review Board of the Aga Khan University Hospital (20192126-5612).

\section{Acknowledgements}

We acknowledge the Department of Surgery of Aga Khan University Hospital Karachi for granting us unlimited access to their database.

\section{References}

1. Mendez-Figueroa H, Dahlke JD, Vrees RA, Rouse DJ. Trauma in pregnancy: an updated systematic review. Am J Obstet Gynecol 2013; 209(1): 1-10. doi: 10.1016/j. ajog.2013.01.021.

2. Hill CC, Pickinpaugh J. Trauma and surgical emergencies in the obstetric patient. Surg Clin North Am 2008; 88(2): 421-40. doi: 10.1016/j.suc.2007.12.006.

3. Schiff MA, Holt VL. Pregnancy outcomes following hospitalization for motor vehicle crashes in Washington State from 1989 to 2001. Am J Epidemiol 2005; 161(6): 50310. doi: 10.1093/aje/kwi078.

4. Tweddale CJ. Trauma during pregnancy. Crit Care Nurs Q 2006; 29(1): 53-67. doi: 10.1097/00002727-20060100000005 .

5. Weiss HB, Sauber-Schatz EK, Cook LJ. The epidemiology of pregnancy-associated emergency department injury visits and their impact on birth outcomes. Accid Anal Prev 2008; 40(3): 1088-95. doi: 10.1016/j.aap.2007.11.011.

6. Kuo C, Jamieson DJ, McPheeters ML, Meikle SF, Posner SF. Injury hospitalizations of pregnant women in the United States, 2002. Am J Obstet Gynecol 2007; 196(2): 161.e1-6. doi: 10.1016/j.ajog.2006.09.015.

7. Karadaș S, Gönüllü $H$, Oncü MR, Kurdoğlu Z, Canbaz
Y. Pregnancy and trauma: analysis of 139 cases. J Turk Ger Gynecol Assoc 2012; 13(2): 118-22. doi: 10.5152/ jtgga.2012.13.

8. Petrone $\mathrm{P}$, Jiménez-Morillas $\mathrm{P}$, Axelrad A, Marini CP. Traumatic injuries to the pregnant patient: a critical literature review. Eur J Trauma Emerg Surg 2019; 45(3): 383-92. doi: 10.1007/s00068-017-0839-x.

9. Zangene M, Ebrahimi B, Najafi F. Trauma in pregnancy and its consequences in Kermanshah, Iran from 2007 to 2010. Glob J Health Sci 2014; 7(2): 304-9. doi: 10.5539/gjhs. v7n2p304.

10. El Kady D, Gilbert WM, Xing G, Smith LH. Association of maternal fractures with adverse perinatal outcomes. Am J Obstet Gynecol 2006; 195(3): 711-6. doi: 10.1016/j. ajog.2006.06.067.

11. Tinker SC, Reefhuis J, Dellinger AM, Jamieson DJ. Epidemiology of maternal injuries during pregnancy in a population-based study, 1997-2005. J Womens Health (Larchmt) 2010; 19(12): 2211-8. doi: 10.1089/ jwh.2010.2160.

12. Mesdaghinia E, Sooky Z, Mesdaghinia A. Causes of trauma in pregnant women referred to Shabih-Khani maternity hospital in Kashan. Arch Trauma Res 2012; 1(1): 23-6. doi: 10.5812/atr.5291.

13. Andersson N, Cockcroft A, Ansari U, Omer K, Ansari NM, Khan A, et al. Barriers to disclosing and reporting violence among women in Pakistan: findings from a national household survey and focus group discussions. J Interpers Violence 2010; 25(11): 1965-85. doi: $10.1177 / 0886260509354512$.

14. Petrone P, Marini CP. Trauma in pregnant patients. Curr Probl Surg 2015; 52(8): 330-51. doi: 10.1067/j. cpsurg.2015.07.001.

15. Weiss HB, Songer TJ, Fabio A. Fetal deaths related to maternal injury. JAMA 2001; 286(15): 1863-8. doi: 10.1001/ jama.286.15.1863.

16. Curet MJ, Schermer CR, Demarest GB, Bieneik EJ, 3rd, Curet LB. Predictors of outcome in trauma during pregnancy: identification of patients who can be monitored for less than 6 hours. J Trauma 2000; 49(1): 18-24. doi: 10.1097/00005373-200007000-00003. 